\title{
Analysis of Quantum Computing and Trellis Coding based on PUM Codes
}

\author{
Vijey Thayananthan \\ Computer Science Department, \\ Faculty of Computing and Information Technology, \\ King Abdul Aziz University, \\ Jeddah 21589, Saudi Arabia.
}

\begin{abstract}
In this paper, we describe the trellis coding where quantum computing (QC) is employed to analyze the coding gain and complexity. According to quantum information theory, quantum bits increase the storage capacity as well as speed. High complex trellis based on partial memory code (PUM) is designed and simulated in digital computers. In order to reduce simulation time for trellis decoding, QC is analyzed with PUM coding. From the theoretical analysis, QC in a quantum computer will be the best option because quantum bits can be stored simultaneously in a given moment of time. From this theory, trellis states, paths, metric calculations are stored in lesser time and space than conventional storing procedures. Therefore, QC certainly will improve the trellis coder performance.
\end{abstract}

\section{General Terms}

In this paper, QC is considered as my general term. Throughout this research, trellis coding analysis is considered with QC.

\section{Keywords}

Trellis coding, QC, complexity, PUM coding, information theory

\section{INTRODUCTION}

Bits are dominated in digital computers, and computations based on digital applications, but quantum bits are involved in quantum computers and computations. It will dominate future applications when digital processing is moving to quantum processing. Binary system provides 0 , and 1 so, digital processing is based on 2 digits. In a quantum system, available quantum bits are more than 2 digits; therefore, quantum processing will take more than 2 digits.

In this research, trellis coding techniques are considered with QC approach. From the theoretical analysis, high-complex trellis designs are easily simulated because quantum bits, and their special properties are flexible to update storage capacities. Trellis designs are constructed using Shannon product theory [5][6], and they are applied in information theory, which deals with source and channel coding.

So far QC is used in both source and channel coding applications, but trellis coding hasn't been considered yet. Under these developments, encoder, decoder and other components have been updated. In order to analyze the trellis coding, PUM trellis coding designs are considered with QC. It is used as an error control coding in communication and digital magnetic recording (storage) channels. It is designed from a combination of block and convolutional coding. Necessary details of PUM coding are explained in section 3 .

\section{QUANTUM COMPUTING}

Quantum computing is the science based on quantum bits calculations. In this computing, nanotechnology takes important roles, which limit according to the applications. It can be used in many complicated algorithms linked with long simulations and high complexities; therefore, QC is targeted in the potential research. It will help to improve the cost, time and energy which are basic factors of innovations. For instance, overall calculations are completed in a fraction of nano second. It means that speed is extremely higher than conventional calculations because properties of quantum bits are different from ordinary bits. However, speed of sound was targeted as a first breakthrough, currently, speed of light is being explored with a number of scientific approaches. Will QC provide any solution for speed of thought? That is the physical limit which should be specified between the speed of light and speed of thought. Whatever calculation in the future quantum computers, it wouldn't go beyond the physical limit. Potential digital computers are constructed with binary building blocks. Electronically, binary 1 is represented as fixed voltage and binary zero is another fixed voltage. These digits can be represented by positive and negative voltages (e.g., +/- 5v). According to the type of applications, values of these voltages are determined. Digital computers are based on these two binary digits. In quantum computers, all computations are based on quantum bits. So, architecture of classical computers is not compatible with quantum computers. This is one of the disadvantages during the quantum computations. Certainly, speed will grow until it reaches the physical limit [9][11][12].

Some of basic features such as adding, shifting etc. are used in quantum computers where executable $\mathrm{QC}$ is employed to do most of the processing. They may not be sufficient to implement quantum computers with specific features. Some selected features such as memory, input, output etc. are important.

\section{TRELLIS CODING}

\subsection{Basic PUM coding}

Here, basic PUM coding is illustrated for QC, but computing complexities during the simulations degrades the performance in digital computers. In order to make a simulation faster than conventional decoders, faster computing is necessary. QC is best option as far as time of processing is concerned during the 
computing. Following equations are used to construct all three types of PUM coding. Construction details are given [1-3].

$$
G_{P U M}=\left[\begin{array}{cccccc}
G_{00}(0) & 0 & 0 & 0 & . . & . . \\
G_{01}(0) & G_{11}(1) & 0 & 0 & . . & . . \\
0 & G_{00}(1) & 0 & 0 & . . & . . \\
0 & G_{01}(1) & G_{11}(2) & 0 & . . & . . \\
0 & 0 & G_{00}(2) & 0 & . . & . . \\
0 & 0 & G_{01}(2) & G_{11}(3) & . & . . \\
0 & 0 & 0 & . & . & .
\end{array}\right]
$$

In equation (1), $G_{00}$ is a generator matrix of the (n, k, $\left.d_{\text {min }}\right)$ basic block code. The $G_{01}$ and $G_{11}$ are the $k_{1} \times n$ matrices respectively $\left(k_{1}<k\right)$. The rank of $G_{11}\left(\operatorname{rank} G_{11}=k_{1}\right)$ because $G_{10}$ is the all-zero $k \times n$ matrix [2][3].

$$
y_{i}=x_{i} *\left[\begin{array}{c}
G_{00} \\
G_{01}
\end{array}\right]+x_{i-1} *\left[\begin{array}{l}
G_{10} \\
G_{11}
\end{array}\right] \quad i=1,2,3 \ldots
$$

In equation (2), vector $x_{i}$ and $y_{i}$ are information and encoded vectors respectively. From equations (1) and (2), a PUM code is modified as $\left(\mathrm{N}, \mathrm{K}, d_{\text {free }}\right)$.

$$
\begin{gathered}
G_{0}=\left[\begin{array}{l}
G_{00} \\
G_{01}
\end{array}\right]=\left[\begin{array}{llllllll}
1 & 1 & 1 & 1 & 1 & 1 & 1 & 1 \\
0 & 0 & 1 & 1 & 1 & 1 & 0 & 0 \\
0 & 0 & 0 & 0 & 1 & 1 & 1 & 1 \\
1 & 0 & 1 & 0 & 1 & 0 & 1 & 0
\end{array}\right] \\
G_{1}^{(8,4,4)}=\left[\begin{array}{l}
G_{10} \\
G_{11}
\end{array}\right]=\left[\begin{array}{llllllll}
0 & 0 & 0 & 0 & 0 & 0 & 0 & 0 \\
0 & 0 & 0 & 0 & 0 & 0 & 0 & 0 \\
1 & 1 & 0 & 0 & 0 & 0 & 0 & 0 \\
0 & 0 & 1 & 1 & 0 & 0 & 0 & 0
\end{array}\right]
\end{gathered}
$$

In order to construct a trellis for $(8,4,4)$ PUM code, above two matrices are needed.

$$
G_{1}^{(8,4,6)}=\left[\begin{array}{l}
G_{10} \\
G_{11}
\end{array}\right]=\left[\begin{array}{llllllll}
0 & 0 & 0 & 0 & 0 & 0 & 0 & 0 \\
1 & 1 & 0 & 0 & 0 & 0 & 0 & 0 \\
0 & 0 & 1 & 1 & 0 & 0 & 0 & 0 \\
0 & 0 & 0 & 0 & 1 & 1 & 0 & 0
\end{array}\right]
$$

Figure 1 shows the trellis for $(8,4,6)$ PUM code constructed from $G_{0}$, and $G_{1}^{(8,4,6)}$ matrices [4- 6].

$$
G_{1}^{(8,4,8)}=\left[\begin{array}{l}
G_{10} \\
G_{11}
\end{array}\right]=\left[\begin{array}{llllllll}
0 & 0 & 0 & 0 & 0 & 0 & 0 & 0 \\
1 & 1 & 1 & 1 & 0 & 0 & 0 & 0 \\
1 & 0 & 0 & 1 & 1 & 1 & 0 & 0 \\
1 & 0 & 1 & 0 & 1 & 0 & 1 & 0
\end{array}\right]
$$

When maximum free distance is used, complexity of the trellis design should be very high.

\subsection{Trellis design for $\mathbf{Q C}$}

Figure 1 provides necessary information about the trellis design of $(8,4,6)$ PUM code. As mentioned in the Table 1 and 2, all the design complexity are shown. When qubits are applied for encoding, 4 of the qubits can be used instead of 4qubits.

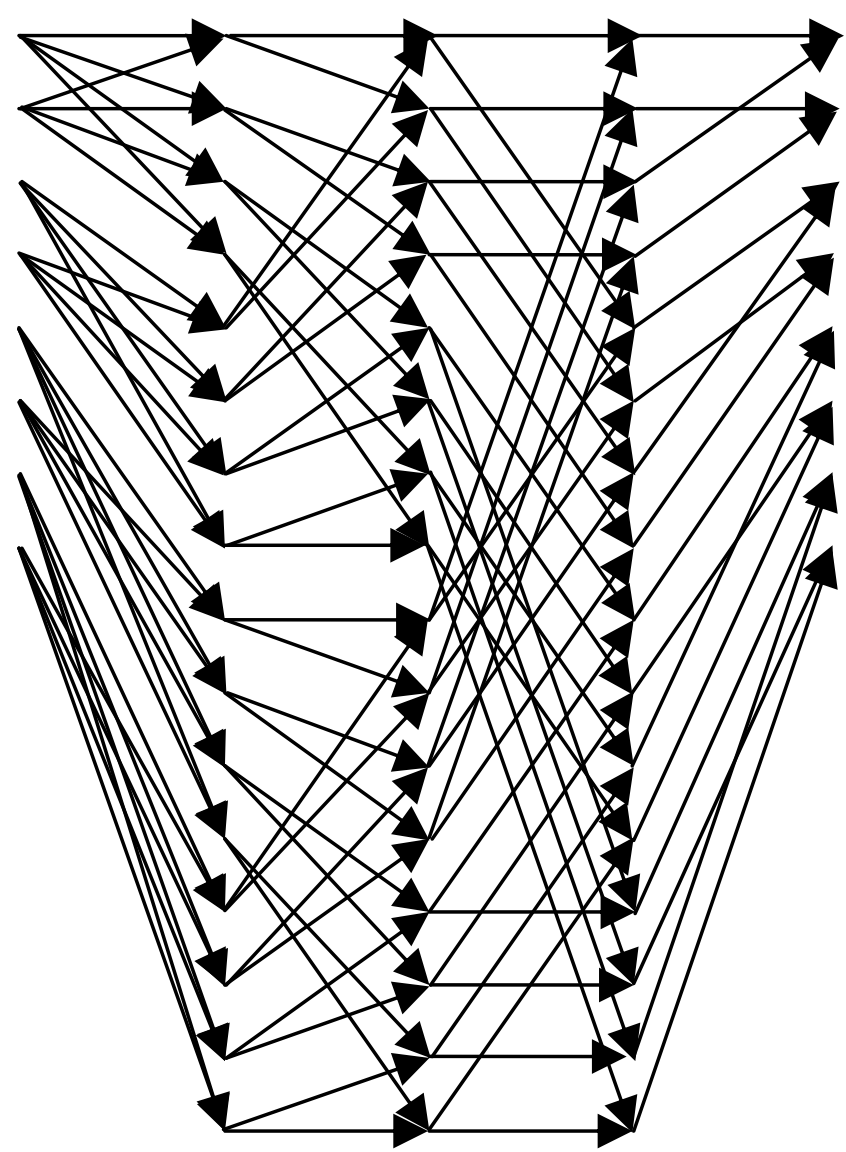

Figure 1: Trellis diagram for $(8,4,6)$ PUM Code [7][8]

\subsection{Comparisons of complexities}

Table 1 shows the complexity analysis for three different coding schemes. This table shows the relationships between the states, columns, branches, additions, comparisons and number of operations. The number of operations is depended on the complexities. If QC is used to Table 1, the complexity can be handled quickly, but processing depends on the number of qubits used in the quantum system.

Table 1. Complexity analysis of $(8,4)$ PUM code

\begin{tabular}{|c|c|c|c|c|c|}
\hline $\begin{array}{c}\text { Trellis } \\
\text { Type }\end{array}$ & States & Col & Branch & Add & Comp \\
\hline$(8,4,4)$ & 32 & 4 & 56 & 56 & 28 \\
\hline$(8,4,6)$ & 64 & 4 & 112 & 112 & 56 \\
\hline$(8,4,8)$ & 112 & 4 & 192 & 192 & 88 \\
\hline
\end{tabular}


Here, same $G_{0}$ matrix is considered to compare the performance of above mentioned codes. The different free distances are applied to the same code considered with following parameters: $\mathrm{N}=8$ and $\mathrm{K}=4$. The number of operations are explained as in Table 2, which shows the total of the additions and comparisons in the $(8,4)$ trellis decoder. According to quantum dynamic theory, encoding vectors will have different data.

Table 2. Comparison of binary and 2qubit operation

\begin{tabular}{|c|c|c|c|}
\hline $\begin{array}{c}\text { Trellis } \\
\text { Type }\end{array}$ & $\begin{array}{c}\text { No. of operation (NOP) } \\
\text { =Add+Comp (binary) }\end{array}$ & $\begin{array}{c}\text { NOP } \\
\text { (2qubit) }\end{array}$ & Gain \\
\hline$(8,4,4)$ & 84 & 21 & 0 \\
\hline$(8,4,6)$ & 168 & 42 & 0.2 \\
\hline$(8,4,8)$ & 280 & 70 & $>0.2$ \\
\hline
\end{tabular}

\subsection{Quantum error correction using trellis}

The error correction for quantum bits are mentioned in many applications with several encoding, and decoding techniques, but trellis coding procedure is considered in this paper. The trellis coding procedures for quantum bits are almost same. When 4 qubits are considered for encoding, 16 different possible bits should be considered.

The decoder program also is written individually for each stage. From the final stage of the final depth metric calculation, the minimum path is selected. In each window, the first depth is taken as corrected path, the remaining next three depths will be moved to the next window. This final path picks up the decoded output which should be one out of 128 possibilities. The each label of the stage has 2 encoded bits. Each state has 16 different weights, which is very useful and easy to compare the path identification. Corresponding path identification, and weight for given information bits produces the decoded output with 1 error correction. Further, the error correction is depended on the free distance as well metric calculation in the trellis. So, it will be increased with the increasing depths [7].

The computer program concerned in this project calculates error performance of decoding programs is simulated with graphical representation. Even, the graphical representation of this trellis is very important for the extra explanation, this program procedure is mainly concerned and written to analyze the results with other conventional decoders. As mentioned in the trellis decoding algorithm, this program is divided into many sub functions which is very easy to modify and extend with other functions. Different noisy signals have been added and compared in this channel. Trellis decoding algorithm increases the error correcting capability and bit error rate (BER) performance when four trellises are used in a single window. Constructed trellis design for $(8,4)$ PUM code has 128 code words represented by trellis path or branches. These 128 code words are used here to select the minimum distance and path.

\subsubsection{Soft decision and quantum computing}

The soft decision is the decoding technique which provides better coding gain when we compare the BER performance. It is proved that overall BER performance is around $2 \mathrm{~dB}$ better than hard decision when we use 4 bits soft decision in the decoder. Quantum bits can be applied as another dimension of soft decision in the decoder as shown in Figure 2. It will certainly improve the BER performance, but decoder complexity will be high. In digital system, when soft decision is implemented in the decoder hardware complexity is considered because it is expensive as well as time consuming. Commercially, BER performance and complexity, are trade-off; therefore, soft decision is limited between 4 bits and 16 bits. Generally, 4 bits soft decision decoder is used in the potential decoder implementations. In trellis decoding, soft decision algorithm for digital system can be implemented easily, but if quantum system is used with same decoder, same algorithm can be used. As far as complexity is concerned in a quantum system, superposition property provides storage capacity simultaneously, but it depends on the qubits. When large qubit is used in the decoder implementation, storage space will grow as mentioned in the Table 3. QC in the trellis decoding will improve the BER performance even complexity is increased. In real quantum system, complexity increases proportionally with storage capacity and qubits. For instance, 168 operations are used in the $(8,4,6)$ decoder as in Table 2 when digital system is used. If two qubits quantum system is used, 168 individual operations (digital) are completed in $168 / 4=42$ simultaneous operations. If four qubits quantum system is used, number of operations even will go down to 11 (10.5 closes to 11$)$.

Figure 2 shows the relationship between 2 dimensional soft decision and 3 dimensional $\mathrm{n}$ qubit computing. The qubits are drawn by thick arrows entangled towards the area of a sphere. From the rectangular plane, soft and hard decision can be calculated for digital system, but $\mathrm{n}$ qubits entangled within the sphere should be considered for a quantum system [10][13][14]. If qubits are on the rectangular plane, calculations are simple as digital system. Full investigation will be considered in my future research.

\section{RESULTS AND COMPARISONS}

In this research, results obtained from the digital computers are compared with quantum computers. Table 1 and Table 2 show that the complexity details when trellis coding simulations are executed in digital computers. Following example provides comparison results computed from QC approach, which takes less memory than the conventional approach.

If 2 quantum bits are allowed, 4 locations are needed to store necessary information mentioned in Table 1 and 2 but quantum system stores all 4 data simultaneously in given a moment.

Digital computer works with binary digits, which mean that 0 or 1 is considered during the single qubit operation. If it is 2 qubits operations, four possible options can be considered one by one. In quantum computers [11], all operations considered simultaneously as mentioned in Table 3 .

Table 3. Storing capacity comparisons

\begin{tabular}{|c|c|c|}
\hline Qbits & $\begin{array}{c}\text { Stores simultaneously in } \\
\text { quantum system }\end{array}$ & $\begin{array}{c}\text { Stores } \\
\text { individually in } \\
\text { digital system }\end{array}$ \\
\hline 1 & $(0 \& 1)$ & $2^{1}=2$ \\
\hline 2 & $(0 \& 1)(0 \& 1)$ & $2^{2}=4$ \\
\hline 3 & $(0 \& 1)(0 \& 1)(0 \& 1)$ & $2^{3}=8$ \\
\hline$:$ & $:$ & $:$ \\
\hline 256 & $(0 \& 1)(0 \& 1) \ldots \ldots . .(0 \& 1)$ & $2^{256}=1.2 \times 10^{77}$ \\
\hline
\end{tabular}


decoding speed against the complexity or number of operations explained in Tables 1 and 2 are considered as a time factor, which increase the iteration and decoding time during the simulations. So, $(8,4,8)$ PUM code simulation time will be very long.

To analyze the results using $\mathrm{QC}$, the prediction results are shown in Table 2 obtained through the simulation using digital system. If it is a real quantum system, simulation results certainly will provide better BER performance. Gain (Table 2) between $(8,4,4)$ and $(8,4,6)$ may be same as digital system, but overall gain will be changed. As I mentioned in section 3.4.1, quantum system is another dimension of soft decision. Gain between the soft and hard decision is already proved. If same proof is applied to a quantum system, BER performance will be improved. It may be called QBER performance rather than BER performance. The QC in a quantum computer is different from digital processing because the principle in quantum mechanics known as superposition changes all these processing.

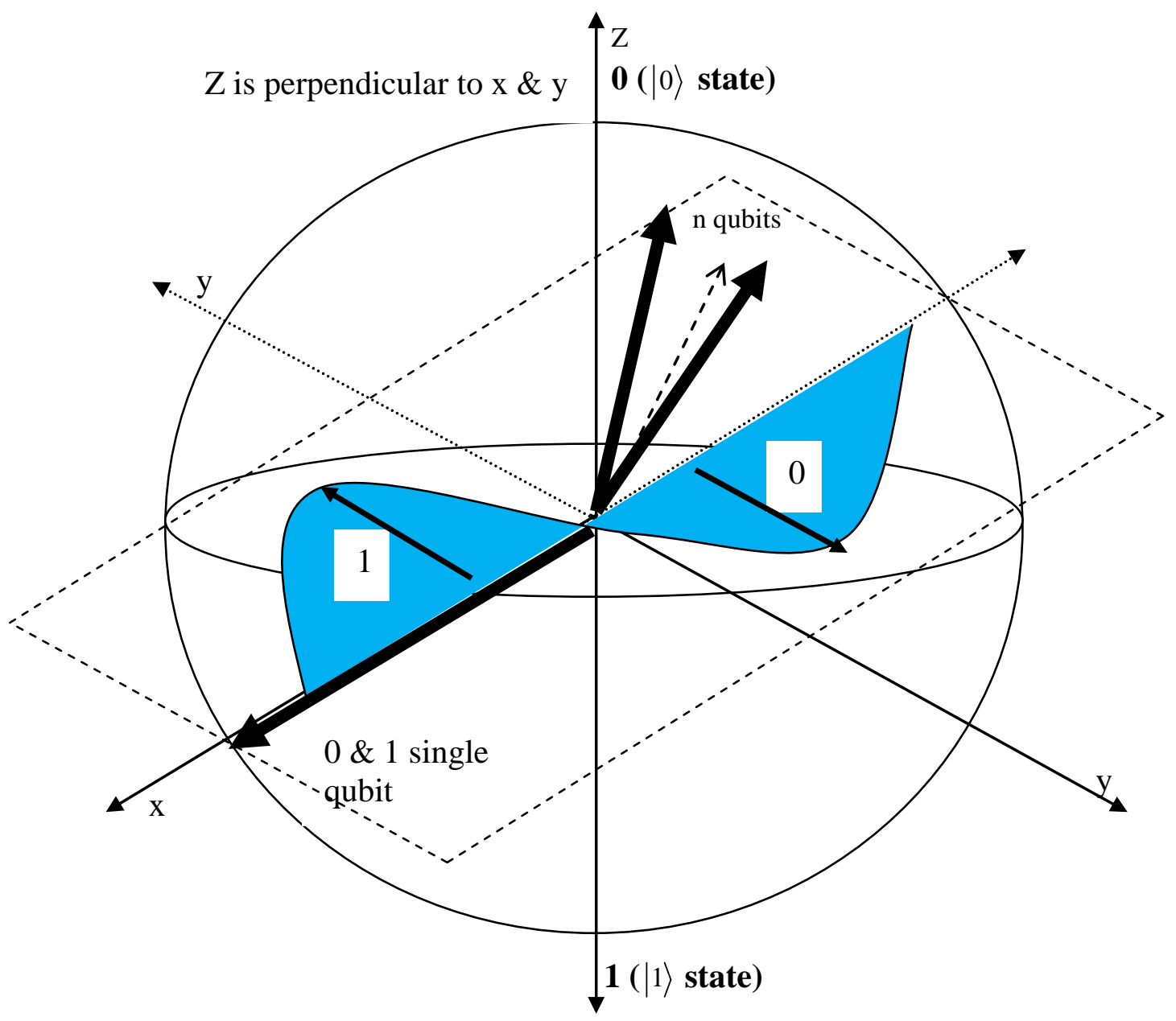

Figure. 2: Hard and soft decision for digital and quantum system

\section{CONCLUSIONS AND FUTUR WORK}

The trellis coding development implemented in digital computer is analyzed with QC and quantum processing. Through this QC procedure, high-complex trellis coding, which include encoding and decoding, can be simulated quickly. Quantum processing is quicker than conventional-digital processing; therefore, speed, time, and cost advantages are easily obtainable in future quantum computers.

Even though no real quantum computer has been completed yet properly, prediction results and analysis of trellis coding have been studied thoroughly. When commercial quantum system is available, number of pending problems will be solved. Specially, cryptography and trellis-coding are expected to use large numbers to make a strong security by 2020 . Again, code breakers are also increased because faster system will break it within the fraction of the second than the conventional system.

\subsection{Future work}

The soft-decision decoder based on QC will be the future research area where qubits and superposition will play a very important role to increase the decoder performance. Through the digital computer, simulation can be completed, but final demonstration should be done in a quantum computer, which hasn't been commercially available yet. 


\section{REFERENCES}

[1] Shraga. I. Bross, "Unit-memory codes for continuous phase modulation in an AWGN channel," IEEE Trans. Inform. Theory, vol.IT-43, pp. 261 - 268, Feb/Mar/April 1995.

[2] Zyablov V. V., Markarian G. S., Portnoy S.L., Balanced codes constructions based on partial unit memory codes, Proceedings of Soviet-Sweden Symposium on Information Theory, Jan 1991, Moscow.

[3] Professor Sidorenko, "Unit Memory/Partial Unit Memory Codes," Lecture Notes 1995.

[4] G. Markarian, B. Honary: 'Trellis decoding technique for block RLL/ECC', IEE Proc.., Oct 1994, vol 141, pp 297 302.

[5] Markarian G., Honary B.,Thayananthan.V. "Trellis Coded Quantisation technique based on partial unit memory codes", Proceedings of the $4^{\text {th }}$ International Symposium on Communication Theory and applications, Ambleside, Lake District, July 1997, pp.56-58.

[6] Vijey Thayananthan, Bahram Honary and Garik Markarian, "Trellis Coded Quantisation Technique Based on Partial Unit Memory Codes", 1998 IEEE International Symposium on Information Theory MIT, August $16^{\text {th }}-21^{\text {st }} 1998$.

[7] Vijey.Thayananthan, Garik Markarian, and Bahram Honary, "DSP Implementation and Design of Trellis Coded Quantisation Technique Based on Partial Unit Memory Codes", IEEE GLOBECOM 98, 812 November 1998.
[8] V. Sidorenko, G. Markarian, and B. Honary. Minimal Trellis Design for Linear Codes Based on the Shannon Product. IEEE Transactions on Information Theory. ISSN 0018-9448. 42(6) pp. 2048-2053. November 1996.

[9] D. A. Lidar, I. L. Chuang, and K. B. Whaley. DecoherenceFree Subspaces for Quantum Computation. Physical Review Letters 81(12):2594\{2597, 21. Sept. 1998.

[10] Schumacher, B., Quantum coding, Phys. Rev. A 51, 27382747 (1995)

[11] E. M. Rains. Nonbinary Quantum Codes. IEEE Transactions on Information Theory, 45(6):1827\{1832, Sept. 1999. See also LANL preprint quant-ph/9703048.

[12] Palmer, J. 2011 Quantum computing device hints at powerful future. Science and technology reporter, BBC News, Dallas. http://www.bbc.co.uk/news/science-environment-12811199.

[13] M. Grassl, A. Klappenecker, and M. R• otteler. Graphs, Quadratic Forms, and Quantum Codes. In Proceedings of the 2002 IEEE International Symposium on Information Theory, page 45, 2002.

[14] A. Ashikhmin and E. Knill. Nonbinary quantum stabilizer codes. IEEE Transactions on Information Theory, 47(7), Nov. 2001. 